\title{
Regeneration in Free Grafts of Normal and Denervated Muscles in the Rat: Morphology and Histochemistry '
}

\author{
BRUCE M. CARLSON AND ERNEST GUTMANN \\ Department of Anatomy, University of Michigan, Ann Arbor, \\ Michigan 48104 and Institute of Physiology, Czechoslovak \\ Academy of Sciences, Prague 4, KRC, Czechoslovakia
}

\begin{abstract}
Intact soleus and extensor digitorum longus muscles in the rat were freely grafted to the contralateral leg after either no preliminary treatment or 14 days prior denervation. Normal muscle grafts during the first week were characterized by a central zone of degenerating original muscle fibers (disappearing by 7-9 days) and a peripheral zone, containing regenerating muscle as well as small numbers of surviving original muscle fibers. A radial gradient of regeneration was established, with more mature muscle at the periphery and less mature muscle toward the center. Denervated grafts were characterized by rapid degeneration (within 2-3 days) of original muscle fibers in the central area, rapid appearance of regenerating muscle fibers (e.g., cross striations by 5 days) with uniform levels of differentiation throughout the graft and larger numbers of surviving original muscle fibers at the periphery. During the first week, stages of muscle differentiation in denervated grafts were attained 1-2 days earlier than comparable stages in normal grafts. Later stages of muscle differentiation were similar in both types of grafts. Histochemical studies revealed a loss of enzyme activity (phosphorylase, ATPase and SDH) in the center of early (2-4day) normal and denervated grafts. Denervated grafts, however, possessed a thicker peripheral rim of enzymatically active surviving muscle fibers than normal grafts. In both types of grafts the old muscle fibers in the center were replaced by enzymatically active regenerating muscle fibers which stained uniformly (ATPase) until 30 days. By 60 days a mixed fiber pattern had developed. Muscle spindles were found within the grafts.
\end{abstract}

Until recent years, attempts at freely grafting entire mammalian skeletal muscles have met little success. Commonly such grafts are resorbed and replaced by a cord of dense fibrous connective tissue (Thompson, '71).

In the past decade Studitsky and his associates (Bosova, '62; Studitsky and Bosova, '60; Studitsky and Zhenevskaya, '67; Zhenevskaya, '68) have demonstrated that entire muscles can be freely grafted if they have been denervated prior to grafting. Denervation from 10-15 days prior to transplantation represents a particularly favorable preparatory interval. Denervation has been postulated to reduce otherwise intact skeletal muscle to a "plastic condition," characterized by a shift to an anerobic metabolic state, a proliferation of nuclei, an increase in RNA and a decreased requirement for oxygen (Studitsky et al.,
'63). Thompson ('71) has successfully employed the free grafting of previously denervated muscles for the treatment of unilateral facial paralysis in humans.

Although the free grafting of unprepared whole muscles in large rats meets with little success (Carlson, '72), it has been reported that in younger animals prior denervation is not a precondition for success in such operations (Zhenevskaya et al., '65). In mice the entire lateral head of the gastrocnemius has also been transplanted without preliminary denervation (Laird and Timmer, '66; Laird-Rolston, '70).

Relatively little is yet known about the

Received Sept. 23, '74. Accepted Mar. 4, '75.

1 Supported by grants from the Muscular Dystrophy Associations of America and a scientific exchange between the Academies of Sciences of the United States and Czechoslovakia. Reported earlier in abstract form (Carlson and Gutmann, '73). 
properties of denervated muscle which enable it to survive free grafting, and much remains to be discovered about the means by which grafted muscles become re-established as functional entities. Studitsky and Zhenevskaya ('67) and Zhenevskaya (' 68, '74) have pointed out that regenerative processes are prominent in transplanted muscles.

After the demonstration of a return to nearly normal contraction times in regenerating minced gastrocnemius muscles of the rat (Carlson and Gutmann, '72), the present authors planned to use free grafting as an experimental model to study the return of function in regenerating fast and slow muscles of the rat. It soon became apparent that this model was more complex than had been originally anticipated because of the survival of a few original muscle fibers at the periphery of the muscle as well as the regeneration of new ones. This report will describe the histological and histochemical properties of normal and denervated fast and slow muscles in the rat following free grafting.

\section{METHODS}

The preliminary morphological survey was conducted upon 30 male SpragueDawley rats (Spartan Farms, East Lansing, Michigan), and the histochemical studies were carried out upon 100 male Wistar rats from the colony at the Institute of Physiology in Prague.

The denervation operation was performed on the left hind leg of 1-month old rats (approximately $60 \mathrm{gm}$ ). Following anesthetization of the rat with ether or $\mathrm{Na}$ pentobarbital, the left sciatic nerve was tightly tied with 6-0 silk thread (Ethicon) as it passed posteriorly around the gluteus maximus muscle and was severed below the ligature. This method is routinely used for long term limb denervations, and reinnervation of the left limbs was never seen in these experiments.

Muscle autotransplantation was accomplished by severing the tendons of origin and insertion of the left soleus (SOL) or extensor digitorum longus (EDL) muscle and removing the muscle intact from the left limb. Following complete removal of the corresponding muscle in the right limb, the left muscle was placed into the bed formerly occupied by the right muscle. The tendons of origin and insertion of the transplanted muscles were connected to the corresponding tendon stumps in the right limb with 7-0 silk sutures. Vascular anastomoses were not attempted. In the preliminary experiments the right nerve stump was sutured to the muscle. In all subsequent experiments this procedure was abandoned because equally successful innervation was obtained by carefully placing the long nerve stump near its normal site of entry into the muscle. Postoperatively the animals were treated with terramycin (in the drinking water for 7 days) or penicillin $(10,000-20,000$ units in a single I.P. injection). Only one transplanted muscle in these experiments became infected and necrotic.

\section{Preliminary morphological series}

In 25 Sprague-Dawley rats the left SOL was denervated for 14,23 or 28 days and then grafted in place of the right SOL muscle. The normal SOL was similarly transplanted in five additional animals. The muscles were removed from 2-112 days after transplantation. They were weighed, fixed in Bouin's and sections were stained in Ehrlich's hematoxylin and eosin, Heidenhain's aniline blue or Hsu's ('71) modification of Palmgren's ('60) silver stain for nerve fibers.

\section{Histochemical series}

Selected transplants, upon which contractile properties had previously been determined (Carlson and Gutmann, '75) were dipped in talcum powder and immediately frozen in liquid nitrogen. The muscles were cut transversely into $10 \mu \mathrm{sec}-$ tions in a cryostat. The sections were stained for myofibrillar adenosine triphosphatase (ATPase) activity (Padykula and Herman, '55) at pH 9.4, using the modification of Guth and Samaha ('70); for succinic dehydrogenase (SDH) activity (Nachlas et al., '57) and for phosphorylase (Ph) activity (Takeuchi and Kuriaki, '55).

\section{RESULTS}

\section{Histology}

Normal soleus grafts. Early transplants were characterized by a large central area composed of ischemic muscle fibers and a 
thin peripheral rim (several hundred microns thick) containing degenerated and regenerating muscle as well as scattered intact mature muscle fibers, apparently survivors of the grafting procedure (figs. 1, 2 ). Two processes predominated in normal grafts during the first week after transplantation. One was the progressive shrinking of the central area of ischemic muscle fibers, and the other was the concomitant expansion of the peripheral zone of regenerating muscle fibers.

In the peripheral zone, those muscle fibers which did not remain intact became invaded by large numbers of macrophages and underwent sarcolysis. Myoblasts appeared within the basement membranes of these degenerated fibers and formed myotubes. The first myotubes appeared in the periphery, starting at 3-4 days. Sarcoplasmic removal of original muscle followed by regeneration of new muscle fibers progressed centripetally, and each day muscle fibers closer to the center of the graft were sarcolysed by invading macrophages. Thus during the first week a radial gradient of differentiation was established, with surviving original muscle fibers and the oldest regenerating fibers seen most peripherally, and successively less mature regenerating muscle fibers being found toward the center of the graft. By the early part of the second week (7--9 days) the central area of ischemic original muscle fibers had completely disappeared. Except for the presence of the few surviving original muscle fibers at the periphery, the overall histological reaction of a normal muscle graft was similar to that of a regenerating minced muscle (Carlson, '72).

By 10 days the grafts were thin. Most of their mass was occupied by young regenerating muscle fibers, many of which still possessed central nuclei. Sections stained by the Palmgren technique revealed only the earliest ingrowth of nerve fibers into the grafts.

Muscles examined 30 days after transplantation were composed primarily of thin striated muscle fibers along with areas of adipose and dense fibrous connective tissue (fig. 3). Nerve fibers were present within the transplants.

Denervated soleus grafts. Previously denervated muscle grafts differed from normal grafts in several ways. A major difference was the rapid rate of disappearance of the central regions of ischemic original muscle fibers - normally by three days. In contrast to the radial gradient of regeneration established in normal grafts, denervated grafts were characterized by regenerating muscle fibers possessing roughly the same degree of maturity throughout the muscle.

Denervated grafts possessed a somewhat thicker peripheral rim of surviving muscle fibers than did normal grafts. Because of their prior denervation, these muscle fibers were thinner than those surviving within normal grafts.

The early stages of regeneration in denervated grafts were accelerated in comparison with those in normal grafts. By three days (fig. 4) almost all of the sarcoplasm of the ischemic muscle fibers had been removed, and within the basement membranes were large numbers of late myoblasts which, by their morphology, were just about to fuse or had already begun to fuse into multinucleated myotubes. Numerous macrophages were present throughout the graft (fig. 4) instead of being concentrated primarily at the junction between the regenerating and ischemic zones as in normal grafts.

In the fourth day after transplantation, large areas of the grafts were already occupied by parallel bundles of early myotubes (fig. 5). By five days a large percentage of the myotubes contained cross-striations although much of the cytoplasm was still basophilic. Regenerating muscle fibers in 6-day transplants had lost their cytoplasmic basophilia, and peripheral migration of the nuclei had begun. By this stage it was not easy to distinguish newly regenerating muscle fibers from persisting old ones, which were still denervated. After the first week, the transplanted muscles contained a uniform population of maturing muscle fibers (fig. 6). Nerve fibers reentered the grafts at the end of the second week.

In the oldest grafts examined (112 days), the muscle fibers had attained nearly normal diameters, and they were histologically normal in most respects (fig. 7). The main deviation from normal 
was the persistence of central nuclei in many of the mature muscle fibers (fig. 8).

\section{Histochemical findings}

Normal EDL grafts. A characteristic histochemical change in grafts of normal muscle was the rapid loss of phosphorylase $(\mathrm{Ph})$ activity. Two days after grafting $\mathrm{Ph}$ could be found only in a very thin rim at the periphery (fig. 9). The thickness of the margin of active fibers increased by four and seven days after transplantation (fig. 10), and at 30 days the regenerating fibers in the interior of the muscle were increasing in $\mathrm{Ph}$ activity (fig. 11). After two months a mixed pattern of fibers with respect to $\mathrm{Ph}$ activity was observed. SDH activity remained low for a considerable period, but ultimately fibers with different levels of activity appeared.

There was a reduction of ATPase activity in the central ischemic fibers of early transplants, but in the same grafts activity remained high in peripheral fibers which had apparently survived. In some peripheral regions a mixed pattern of ATPase remained. Thirty days after transplantation the ATPase reaction of the muscle fibers within the graft was still quite uniform and many dilated blood vessels were seen. At later stages the recovery of normal fiber diameter as well as the mixed staining reaction for ATPase had occurred (fig. 12). Type I, type II and intermediate fibers were present.

Denervated EDL grafts. As in free grafts of the normal EDL, there was a loss of $\mathrm{Ph}$ activity in the central part of the muscle, but the peripheral rim of active fibers was broader than in normal muscle grafts (fig. 13). In denervated transplants occasional areas of $\mathrm{Ph}$ activity were seen in the central portions at 2-4 days. With the rapid regeneration of muscle fibers in the denervated EDL, $\mathrm{Ph}$ activity reappeared quickly both at the periphery and in the central portions of the grafts. In the late periods after transplantation, $\mathrm{Ph}$ activity of muscle fibers was mixed, but there was a preponderance of fibers with high activity. Muscle spindles with fibers staining heterogeneously for $\mathrm{Ph}$ have been seen in older regenerates (fig. 14).

ATPase activity was generally reduced two days after transplantation, but scat- tered hypertrophic fibers with high activity were found at the periphery. By seven days the interior of the transplant was filled with early regenerating muscle fibers, and these fibers, with small diameters, showed equal, low levels of activity. Thirty days after transplantation the diameters of the muscle fibers had increased considerably, but the ATPase staining reaction was still uniform (fig. 15). The later stages of differentiation of denervated EDL grafts resembled those of the normal EDL grafts (fig. 16), and except for the patchy distribution of fiber types, they did not differ greatly from those of normal muscle ( $\mathrm{cf}$. fig. 1 of Melichna and Gutmann, '74).

SDH activity in denervated EDL grafts was initially low, but it increased progressively following the seventh day after transplantation. At seven days patches of fibers with higher SDH activity than the surrounding fibers were seen in the periphery. These were probably original fibers which survived the transplantation procedure. In long term transplants a mixed fiber pattern was established (fig. 17) although as with the ATPase reaction, the fiber types were not so evenly distributed as those of a normal EDL muscle (cf. fig. 4 of Melichna and Gutmann, '74).

Normal SOL grafts. Two days after transplantation, normal SOL grafts were devoid of $\mathrm{Ph}$ activity in all areas except for a thin peripheral rim of fibers which were highly reactive. As the regenerating muscle fibers in the central area matured, a return of $\mathrm{Ph}$ activity was seen. Even after several months, scattered fibers of the grafts demonstrated considerably higher levels of $\mathrm{Ph}$ activity than are normally found in the SOL muscle.

The ATPase of normal SOL grafts during the early days after transplantation was also decreased in the central areas whereas the peripheral muscle fibers maintained a near normal pattern of ATPase activity. By 14 days the thin regenerating muscle fibers in the center of the graft exhibited a fairly uniform staining pattern. In late stages the characteristic mixed pattern, with a predominance of type I fibers, was re-established (fig. 18).

In normal SOL grafts SDH activity remained low 30 days after transplantation, and even at 90 days the activity of SDH 
was relatively low although it was higher than in EDL grafts of the same age.

Denervated SOL grafts. Like the denervated EDL, more peripheral fibers of early denervated SOL grafts retained Ph activity than those in normal grafts. Even in the central parts of 2-day denervated grafts, fibers with some degree of $\mathrm{Ph}$ activity were seen whereas in normal SOL grafts there was a complete loss of $\mathrm{Ph}$ activity in the central area. In general, there was a greater degree of $\mathrm{Ph}$ activity in denervated grafts than in normal grafts during the first week. The differences between the two types of grafts were not maintained during the later stages.

Two day denervated grafts showed a mixed pattern of ATPase activity within the original muscle fibers less restricted to the peripheral fibers than normal grafts. By 30 days a mixed staining pattern had begun to emerge in the regenerating muscle fibers, with most fibers having lower activity levels than those of denervated EDL grafts (fig. 19).

In summary, the histochemical studies have revealed a sharp decrease in enzymatic activity, particularly of $\mathrm{Ph}$, in the central regions of early normal EDL and SOL grafts. The decrease in the EDL is more pronounced than in the SOL. Denervated grafts, particularly the SOL, show a greater proportion of enzymatically active fibers scattered more centrally within the grafts. Mature levels of enzymes are progressively built up in regenerating muscle fibers within the grafts so that mixed fiber patterns are found in 2-3-month transplants. The patterns of enzymatic activity of older grafts, however, are abnormal in distribution, with a greater tendency toward clumping of similar fiber types. Intrafusal muscle fibers with a mixed pattern of enzymatic activity have been found in free grafts.

\section{DISCUSSION}

This work confirms the earlier reports (Studitsky and Zhenevskaya, '67; Zhenevskaya et al., '65) that a major factor in the successful free grafting of skeletal muscle is a massive regeneration of new muscle fibers within the graft. Morphologically there appears to be also a small contribution of surviving peripheral muscle fibers.
Because of the small size of the muscles used in this experiment, the differences between previously denervated grafts and normal grafts were not so great as those seen in grafts of larger muscles. In free autografts of the normal gastrocnemius muscle in rats, Carlson ('72) reported that a wave of polymorphonuclear leukocytes sweeps through the graft, leaving in its wake empty endomysial tubes. These are replaced by dense masses of collagenous tissue, and seldom is any evidence of muscle regeneration seen. Thompson ('71) has summarized the results of other investigators who have noted the same fate of unprepared skeletal muscle transplants. The work of Zhenevskaya et al. ('65), Laird and Timmer ('66) and Laird-Rolston ('70) as well as the present study has shown that if a muscle is small enough, considerable regeneration will occur in normal muscle grafts.

The major morphological differences between normal and denervated grafts of small muscles occur during the first week after transplantation. Denervated grafts show a very rapid and uniform cycle of degeneration of old muscle fibers and regeneration of new ones whereas normal grafts reacted more slowly and displayed a centripetal gradient of degeneration and regeneration. Histochemically, there was greater remaining enzymatic activity during the early days after transplantation in denervated grafts than in normal ones, but in both cases the pattern of activity suggests severe ischemia in the central regions of the grafts and the probable survival of fibers through diffusion of nutrients in the peripheral rim.

The acceleration of early regenerative phases in muscle denervated prior to trauma has been noted also by investigators studying minced muscle regeneration (Hsu, '71; Yeasting, "69). A likely explanation is based upon the reports of Lee ('65), Hess and Rosner ('70) and Aloisi ('70) that in the days following denervation there is a pronounced increase in mononuclear cells located between the sarcolemma and the basement membrane of the muscle fiber. If these mononuclear cells are potentially myoblastic, the first couple of days after transplantation could then be devoted to the immediate activation of these cells 
rather than the recruiting of a quantitatively sufficient supply of myoblasts.

Neither the histological nor the histochemical characteristics of the maturing transplants differed remarkably from those of other regenerating mammalian muscle except that the completeness of regeneration was considerably greater than that found in most other systems of muscle regeneration. Histochemical maturation of the regenerating muscle fibers follows histological maturation, for the muscle fibers are histologically mature at the end of the first month, but yet have not developed distinct histochemical fiber types.

The presence of muscle spindles in grafts is noteworthy. These are not seen in minced muscle regenerates from older animals (Carlson, '72; Zelená and Sobotková, '71). Spindles seen in early grafts are of a uniform histochemical pattern and only later do the fibers develop mixed staining reactions. This may indicate the regeneration of intrafusal fibers with a recovery of mixed muscle fiber pattern or it may possibly be a reaction to the grafting procedure of spindles already present.

Little is yet known, particularly at the chemical level, about factors that account for the different reactions to transplantation of normal and denervated muscles. At the gross level, size alone is one factor. Because of the reduction in diameter of individual denervated muscle fibers, denervated grafts as a whole are thinner than their normal counterparts. This would allow a quicker return of a vascular supply to the inner fibers of the graft and lessen the chance of their death due to ischemia. A histological observation that merits further attention is the rapid degeneration of old muscle fibers throughout the previously denervated graft during the first couple of days following transplantation. It is known from studies on minced muscle regeneration (Carlson, '68, '72) that regeneration of new muscle fibers does not progress in areas where the sarcoplasm of the original muscle fibers remain intact. Possibly prior denervation renders the muscle fibers less stable in the ischemic environment of the center of the early transplants, and they degenerate more quickly. This, along with an ability of myoblastic precursors to sur- vive temporarily in an avascular environment, could possibly account for the difference in the early reactions of denervated and normal grafts to transplantation.

\section{LITERATURE CITED}

Aloisi, M. 1970 Patterns of muscle regeneration. In: Regeneration of Striated Muscle and Myogenesis. A. Mauro, S. A. Shafiq and A. T. Milhorat, eds. Excerpta Medica, Amsterdam, pp. 180-193.

Bosova, N. N, 1962 Free autoplastic transplantation of whole muscles (Russian). Byull. Exp. Biol. Med., 53(3): 88-92.

Carlson, B. M. 1968 Regeneration of the completely excised gastrocnemius muscle in the frog and rat from minced muscle fragments. J. Morph., 125: 447-471.

1972 The Regeneration of Minced Muscles. S. Karger AG, Basel.

Carlson, B. M., and E. Gutmann 1972 Development of contractile properties of minced muscle regenerates in the rat. Exp. Neurol., 36: 239-249.

1973 Regeneration in freely transplanted intact muscles of the rat. Anat. Rec., 28: 284 (abstract).

- 1975 Regeneration in grafts of normal and denervated rat muscles. Contractile properties. Pflüger Arch., 353; 215-225.

Guth, L., and F. J. Samaha 1970 Procedure for the histochemical demonstration of actimyosin ATPase. Exp. Neurol., 28: 365-367.

Hess, A., and S. Rosner 1970 The satellite cell bud and myoblast in denervated mammalian muscle fibers. Am. J. Anat., 129: 21-40.

Hsu, L. 1971 The role of nerves in the regeneration of minced muscle in adult anurans. Doctoral Dissertation, University of Michigan, Ann Arbor.

Laird, J. L., and R. F. Timmer 1966 Transplantation of skeletal muscle into a host with muscular dystrophy. Texas Rep. Biol. Med., 24: $169-179$.

Laird, Rolston, J. L. 1970 Further studies on the orthotopic transplantation of skeletal muscle. Texas Rep. Biol. Med., 28: 97-104.

Lee, J. C. 1965 Electron microscopic observations on myogenic free cells of denervated skeletal muscle Exp. Neurol., 12: 123-135.

Melichna, J., and E. Gutmann 1974 Stimulation and immobilization effects on contractile and histochemical properties of denervated muscle. Pflügers Arch., 352: 165-178.

Nachlas, M. M., K. C. Tsou, E. Souza, C. S. Cheng and A. M. Seligman 1957 Cytochemical demonstration of succinic dehydrogenase by the use of a new p-nitrophenyl substituted ditetrazole. J. Histochem. Cytochem., 5: 420436.

Padykula, H. A., and E. Herman 1955 The specificity of the histochemical method for adenosine triphosphate. J. Histochem. Cytochem., 3: 170-195. 
Palmgren, A. 1960 Specific silver staining of nerve fibers. I. Technique for vertebrates. Acta Zool., 41: 239-265.

Studitsky, A. N., and N. N. Bosova 1960 Development of atrophic muscular tissue in conditions of transplantation in place of mechanically damaged muscles (Russian). Arkh. Anat. Gist. Embriol. 39 (12): 18-32.

Studitsky, A. N., and R. P. Zhenevskaya 1967 Theory and Practice of the Auto- and Homotransplantation of Muscles. Publ. House "Nauka," Moscow.

Studitsky, A. N., R. P. Zhenevskaya and O. Rumyantseva 1963 The role of neurotrophic influences upon the restitution of structure and function of regenerating muscles. In: The Effect of Use and Disuse in Neuromuscular Functions. E. Gutmann and P. Hník, eds. Publ. House Czecholovak Acad. Sci., Prague, pp. 7181.

Takeuchi, T., and H. Kuriaki 1955 Histochemical detection of phosphorylase in animal tissues. J. Histochem. cytochem., 4: 153-161. Thompson, N. 1971 Autogenous free grafts of skeletal muscle. Plastic Reconstr. Surg., 48: 11-27.

Yeasting, R. A. 1969 The effect of the nerve supply on the regeneration of minced skeletal muscle in the mouse. Doctoral Dissertation, University of Louisville, Louisville.

Zelená, J., and M. Sobotková 1971 Absence of muscle spindles in regenerated muscles of the rat. Physiol. bohemoslov., 20: 433-439.

Zhenevskaya, R. P. 1968 Transplantation of skeletal muscles in animals (Russian). Uspekh. Sovrem. Biol., 65: 133-143.

1974 Neurotrophic Regulation of the Plastic Activity of Muscular Tissue (Russian). Izdatel. Nauka, Moscow.

Zhenevskaya, R. P., O. N. Rumyantseva, I. L. Novoselova and E. V. Proshlyakova 1965 Regenerative processes in a transplant of unprepared muscle of young rats (Russian). $\mathrm{Zh}$. Obsch. Biol., 26: 569-576. 
PLATE 1

EXPLANATION OF FIGURES

1 Three-day normal SOL graft. Muscle fibers in the center of the graft (right) are typically ischemic, but have not yet undergone extensive sarcolysis. At the periphery the sarcoplasm of most muscle fibers has undergone extensive degeneration, and in some areas early regenerative changes are beginning. $H \& \mathrm{E} . \times 37.12$.

2 High power view of the periphery of the same regenerate as figure 1 , showing histologically intact muscle fibers interspersed among muscle fibers in advanced states of degeneration. Note the large number of macrophages in the degenerating muscle. H \& $\mathrm{E} \times 203$.

3 Thirty-day normal sol graft. The thin graft contains regenerated muscle fibers, bands of connective tissue and occasional fat cells. H \& E. $\times 63.8$.

4 Three-day denervated (23 days) SOL graft. A band of original denervated muscle fibers remains intact at the periphery (left), but in the interior of the graft (center and right) almost all original muscle fibers have degenerated extensively and are being replaced by populations of myoblasts. Compare with 3-day normal graft (fig. 1). H \& E. $\times 34.8$. 

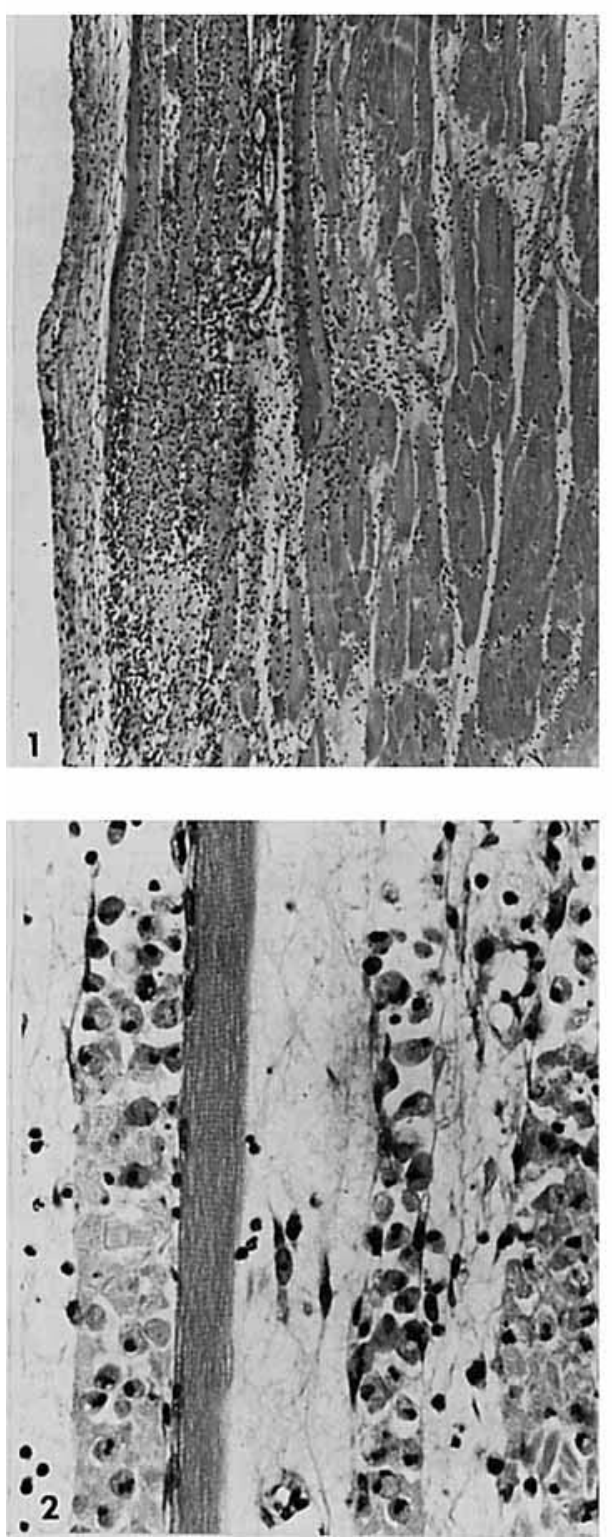
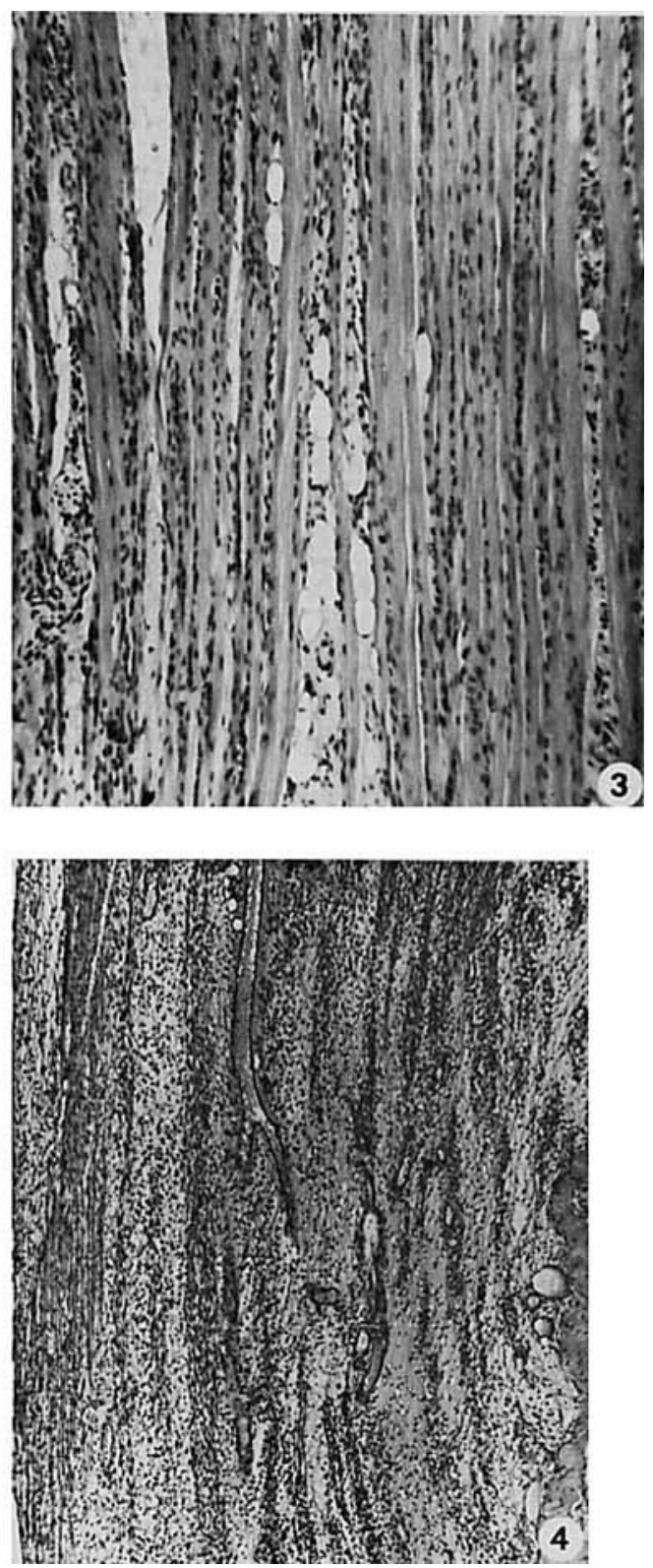
PLATE 2

EXPLANATION OF FIGURES

5 Four-day denervated (23 days) SOL graft. The entire graft, except for the peripheral rim, is filled with early myotubes. $H \& \mathrm{E}, \times 44.08$.

6 Ten-day denervated ( 23 days) SOL graft. The entire graft is filled with a uniform population of young muscle fibers. $\mathrm{H} \& \mathrm{E} . \times 58$.

7 One hundred twelve-day denervated (23 days) SOL graft. Around the transplant is a thick sheath of connective tissue. Cross section. H \& E. $\times 23.2$.

8 Higher power view of the same section as figure 8 , showing a muscle spindle (arrow) and central nuclei in a number of the regenerated muscle fibers. H \& E. $\times 203$. 

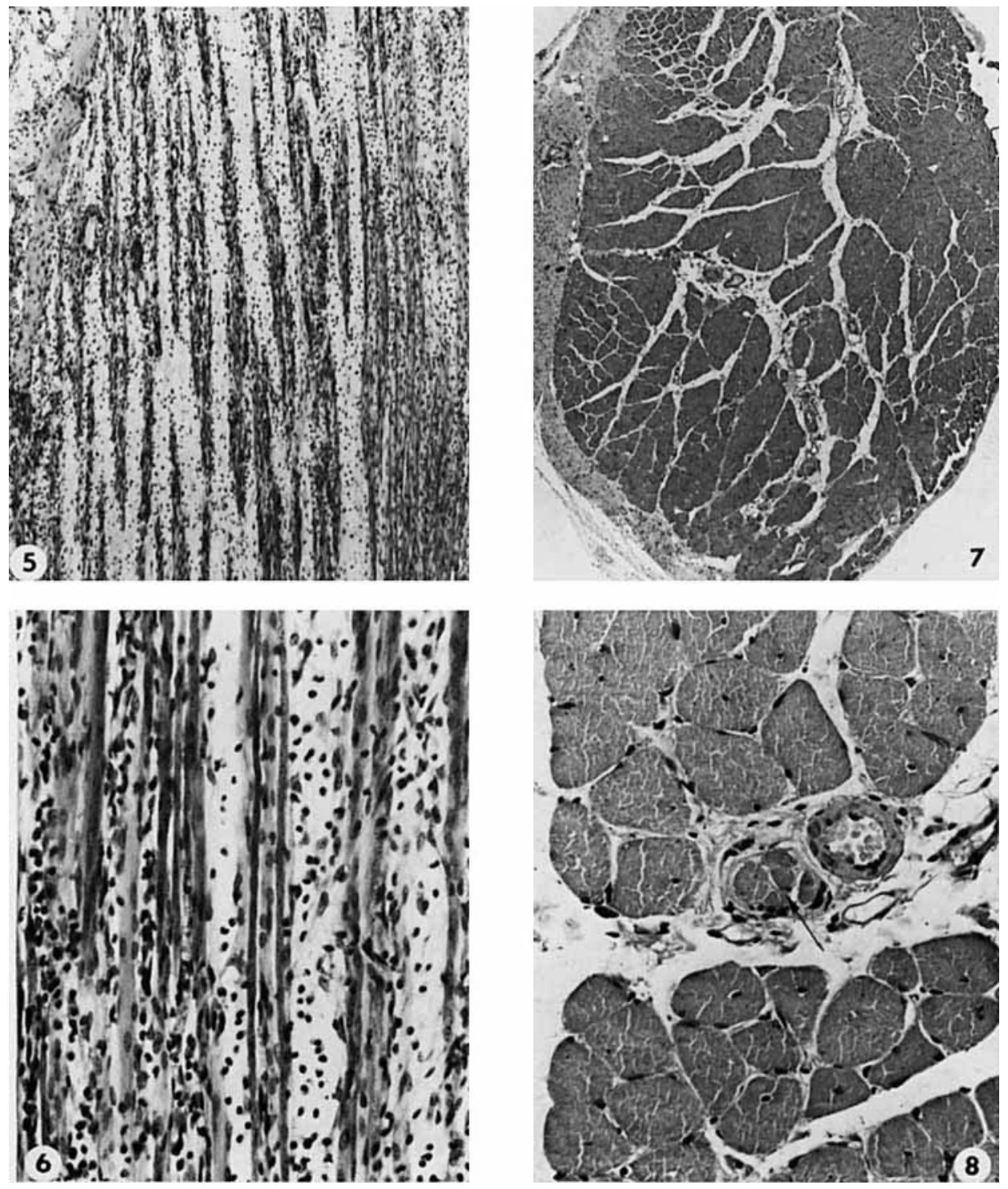
PLATE 3

EXPLANATION OF FIGURES

9 Two-day graft of normal EDL muscle. Only a few of the most peripheral muscle fibers display any $\mathrm{Ph}$ activity. $\times 89.9$.

10 Seven-day graft of normal EDL muscle, A greater number of old muscle fibers at the periphery demonstrate $\mathrm{Ph}$ activity. $\times 89.9$.

11 Thirty-day graft of normal EDL muscle showing increasing $\mathrm{Ph}$ activity in muscle fibers in the interior of the graft. $\mathrm{Ph} . \times 89.9$.

12 Ninety-day graft of normal EDL muscle, demonstrating distinct fiber types. ATPase. $\times 89.9$.

13 Two-day graft of 14-day denervated EDL muscle. A thicker rim of active fibers is present than in normal grafts (fig. 9). $\mathrm{Ph} . \times 89.9$.

14 Sixty-day graft of 14-day denervated EDL muscle, showing a muscle spindle (arrow). Ph. $\times 174$. 

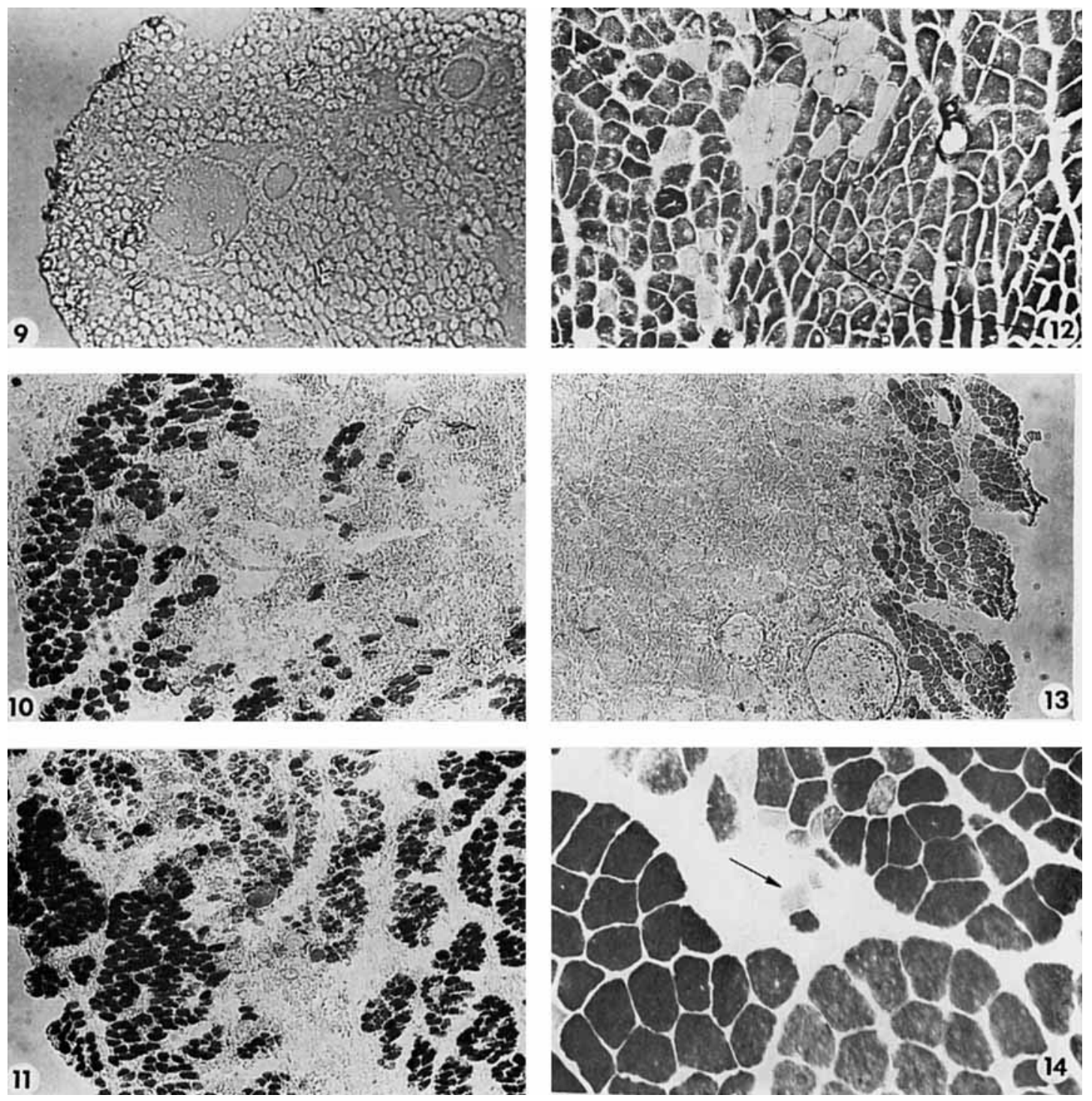
PLATE 4

EXPLANATION OF FIGURES

15 Thirty-day graft of 14-day denervated EDL. The muscle fibers still stain uniformly for ATPase activity. $\times 89.9$.

16 Sixty-day graft of 14-day denervated EDL muscle, showing distinct fiber types. ATPase. $\times 89.9$.

17 Sixty-day graft of 14-day denervated EDL muscle, showing mature pattern of SDH activity. $\times 89.9$.

18 Ninety-day graft of normal SOL muscle. ATPase. $\times 89.9$.

19 Thirty-day graft of 14-day denervated SOL muscle. Slight differences in ATPase activity are beginning to appear. $\times 89.9$. 

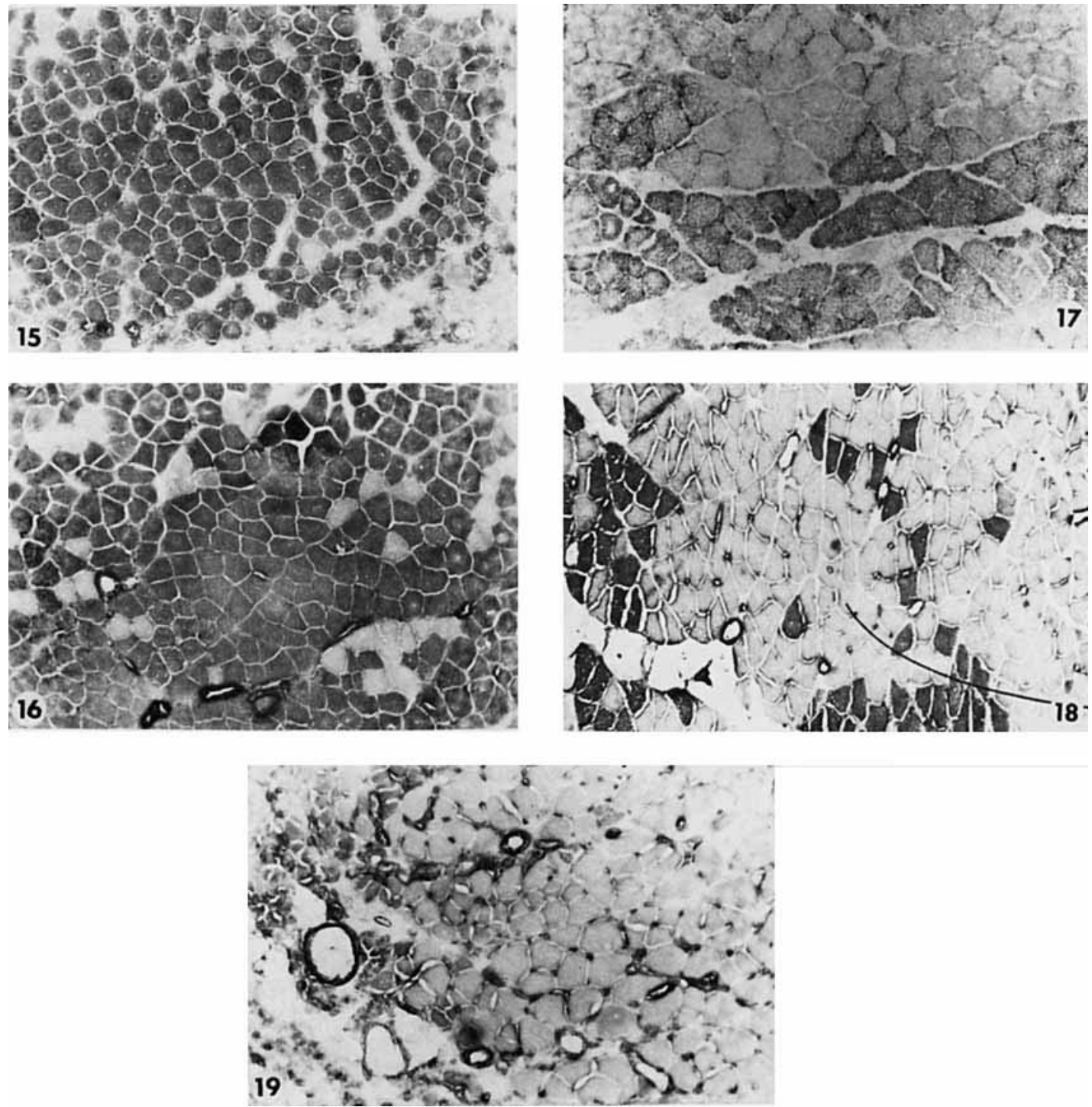